\title{
Edge Detection to Isolate Motion in Adaptive Optics Systems
}

C. Chan

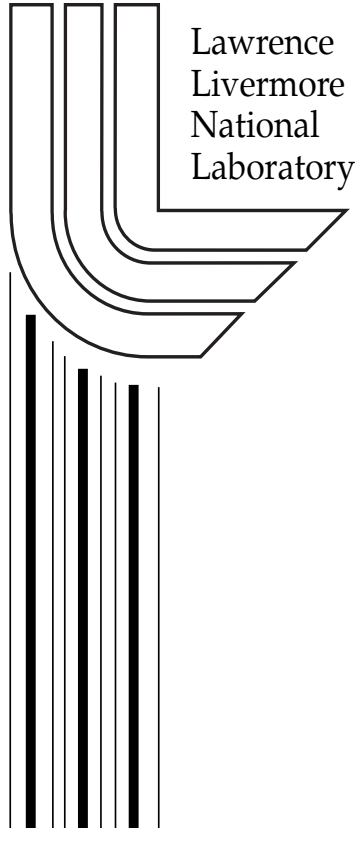

July 11, 2003

National 
This document was prepared as an account of work sponsored by an agency of the United States Government. Neither the United States Government nor the University of California nor any of their employees, makes any warranty, express or implied, or assumes any legal liability or responsibility for the accuracy, completeness, or usefulness of any information, apparatus, product, or process disclosed, or represents that its use would not infringe privately owned rights. Reference herein to any specific commercial product, process, or service by trade name, trademark, manufacturer, or otherwise, does not necessarily constitute or imply its endorsement, recommendation, or favoring by the United States Government or the University of California. The views and opinions of authors expressed herein do not necessarily state or reflect those of the United States Government or the University of California, and shall not be used for advertising or product endorsement purposes.

This work was performed under the auspices of the U.S. Department of Energy by University of California, Lawrence Livermore National Laboratory under Contract W-7405-Eng-48. 


\title{
Edge Detection to Isolate Motion in Adaptive Optics Systems
}

\author{
Carri Chan 7/11/03
}

\section{Introduction}

Adaptive optics uses signal processing techniques and deformable mirrors to minimize image degradation caused by phase abberations. In the case of telescope imaging, the atmosphere causes phase aberrations. In the case of satellite imaging, errors due to the ultra-light-weight characteristics of the primary mirror cause phase aberrations. Scene-based Shack-Hartmann Wave Front Sensing takes the correlation between successive wavelets to determine these phase aberrations. [1]

A large problem with the scene-based approach is that motion, such as a moving car, can cause the correlation of two lenslets to peak, not where the scenes align, but where the moving object in each frame aligns. As such, the continued use of scene-based Wave Front Sensing necessitates successful isolation of moving objects from a stationary background scene. With the knowledge of which pixels are immobile, one should avoid the problem of locking onto a moving object when taking the correlation of two successive frames in time.

Two main requirements of isolation are consistency and efficiency. In this document I will discuss the different edge detection algorithms explored for moving object isolation and how I came to the conclusion that, for our purposes of scene-based Shack-Hartmann WFS, edge detection is too inconsistent to be of any use. Because the Shack-Hartmann lenslets limits us to low resolutions, edge detection that works on higher resolution images will not work on our images. The results of each algorithm will show that with so few pixels per subaperature, edge detection is a poor method of identifying moving objects.

\section{Method}

The purpose of the edge detection is to differentiate between edges that remain constant in a scene from those that move. The tracking of moving edges is done by using a reference mask generated by comparing the set of edges in each subsequent frame in time to the current reference mask. At initialization, the reference mask is set to be the edges in the first frame. As time goes on, the edges that move are removed from the reference mask, while those that don't are left behind. Only the edges that are consistent in both the current frame and the reference mask are maintained in the updated reference. This prevents the edges of moving objects from being stored in the reference mask. By iteratively updating the reference mask, the algorithm identifies the static sections of the scenes.

\section{High-Pass Smoothing Filter}

One edge detection algorithm uses a high-pass boxcar filter via IDL's smooth function. smooth acts as a low-pass filter by taking the average of neighboring pixel values which blurs edges. By subtracting the smoothed image from the original image, the image has been high-pass filtered, leaving the edges highlighted. Edges are defined to be pixels with large high frequency content, determined by a hard-coded threshold. smooth must look at every single pixel in the $\mathrm{nxn}$ image and average over an encompassing block of kxk pixels. If $\mathrm{k}$ were large, on the order of $\mathrm{n}$, this filter would take $\mathrm{O}\left(\mathrm{n}^{4}\right)$ time. However, in order to have a narrower frequency band, only a small, constant $3 \times 3$ block is averaged to find the filtered pixel value, so that this high-pass filter takes $\mathrm{O}\left(9 \mathrm{n}^{2}\right)$ time. 


\section{Ideal High-Pass/Butterworth High-Pass Filters}

Two filters, which are implemented in the frequency domain and should detect edges, are an ideal high-pass filter and a first-order Butterworth high-pass filter. The ideal high-pass filter only passes frequencies above a specified cut-off frequency. The Butterworth filter is a digital approximation of the continuous Gaussian filter in which not all frequencies below the cut-off frequency are minimized uniformly. Each of these filters was implemented with a hard-coded cut-off frequency. This cut-off frequency varied between $1 / 4$ and $3 / 4$ of the sampling frequency in order to determine a cut-off frequency that would detect edges consistently in different images. Filters implemented in the frequency domain can utilize the speed of the FFT, making for efficient edge detection. For an nXn image, filtering takes $O\left(n^{2} \log n\right)$ time, whereas, if these filters were to be implemented in the spatial domain, $n x n$ convolutions would take $\mathrm{O}\left(\mathrm{n}^{4}\right)$ time.

\section{Prewitt/Sobel Masks}

Using edge masks, which are implemented in the spatial domain, is a very popular method of edge detection amongst most digital-image processing books. These masks are convolved with the image to estimate the rate of change between pixel intensities. The higher the rate of change, the more likely there is an edge. While, in general, convolutions in the spatial domain involve more computation than processing images in the frequency domain, this is not the case because the edge masks, instead of being nxn, are of constant, $3 \times 3$, size. Therefore, the use of these edgedetection masks is actually less computationally expensive than the frequency domain filters because they take $\mathrm{O}\left(\mathrm{n}^{2}\right)$ time.

Edge-detection masks developed by Prewitt and a hard-coded threshold defined edges and nonedges. Sobel masks were used separately, in the same manner as the Prewitt masks, to detect edges. Sobel masks are very similar to Prewitt masks. However, by giving more weight to the central pixel, they are claimed to be more robust for noisy images.

\section{$2^{\text {nd }}$ Derivative Zero-Crossing}

While the Prewitt and Sobel masks estimate the $1^{\text {st }}$ derivative of the image intensities, edges can also be characterized by their $2^{\text {nd }}$ derivative. Edges have a $2^{\text {nd }}$ derivative of zero. The onedimensional discrete $2^{\text {nd }}$ derivative is a $2^{\text {nd }}$ difference function and can be expressed by the following equation:

$$
\mathrm{d}^{2} \mathrm{f}(\mathrm{i}) / \mathrm{d}(\mathrm{i})=\mathrm{f}(\mathrm{i}+1)-2 \mathrm{f}(\mathrm{i})+\mathrm{f}(\mathrm{i}-1)
$$

The $2^{\text {nd }}$ derivative can also be calculated in the frequency domain. The following simple relationship between the FFT of an image and the FFT of its $2^{\text {nd }}$ derivative make it easy to implement this filter in the frequency domain:

$$
\square^{2} f=\mathcal{F}^{1}\left[-4 \square^{2}\left(u^{2}+v^{2}\right) \mathcal{F}(f)\right][2]
$$

When the $2^{\text {nd }}$ derivative is converted back into the spatial domain, zero crossings isolate edges. Because this filter is implemented in the frequency domain, it takes $\mathrm{O}\left(\mathrm{n}^{2} \log n\right)$ time.

\section{Results}

Tests of each edge detection algorithm performed on a series of images show that edge detection is an ineffective method to isolate moving objects. The first set of images was of 32x32 "model" images, where edges were a pixel wide and the contrast levels between objects was high. Noisy and shifted versions of the model images were also used. The noise model of this system is Poisson. The pixel in a noisy image is characterized as a Poisson process with its mean and variance equal to the actual pixel value of the noise-free image. On top of the noisy pixel value, read-noise, which is also modeled as a Poisson random variable, is added. One must note that the noise in this system is independent. Each shifted image is a shifted version of the original image. Because each image shift is a randomly chosen subpixel shift, the shift is implemented in the 
frequency domain. For an nxn image, where $S[u, v]$ is the discrete-time Fourier Transform of the shifted image, and $\mathrm{I}[\mathrm{u}, \mathrm{v}]$ is the discrete-time Fourier Transform of the original image, a shift of $\square \mathrm{x}$ and $\square \mathrm{y}$ can be implemented in the frequency domain by the following function:

These "model" images can be seen below.

$$
\mathrm{S}[\mathrm{u}, \mathrm{v}]=\mathrm{I}[\mathrm{u}, \mathrm{v}] \mathrm{e}^{-\mathrm{j} 2 \square\left(\square \mathrm{x}^{*} \mathrm{u}+\square \mathrm{y}^{*} \mathrm{v}\right) / \mathrm{n}}[1]
$$

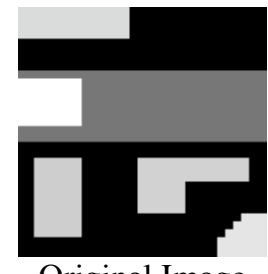

Original Image

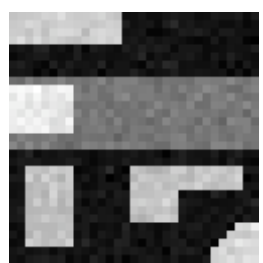

Noisy Image

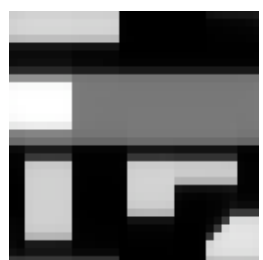

Shifted image

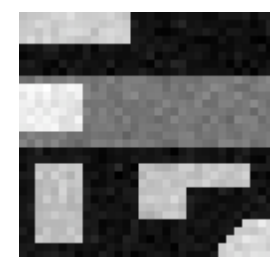

Shifted \& Noisy Image

The next set of images was composed of real imaging data. Unlike the model data, edges were not necessarily a single pixel wide. In fact, some could have been multiple pixels wide, while other edges could have been in between pixels. Using histogram binning, I down sampled a $256 \times 256$ image into $32 \times 32$ pixels. This $32 \times 32$ image most accurately depicts the image a CCD camera would detect because it takes the average of neighboring pixel values to emulate the total amount of light each sensor detects. To that 32x32 image, noise and subpixel shifts were added to it, just as had been done with the model data. These images can be seen below

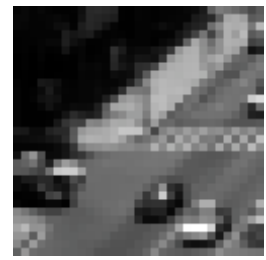

Original Image

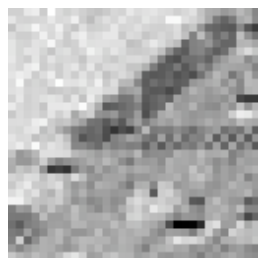

Noisy Image

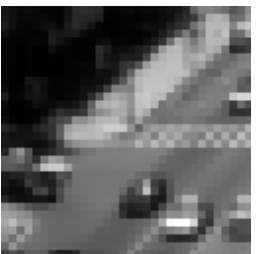

Shifted image

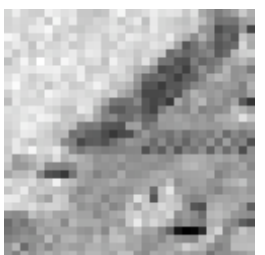

Shifted \& Noisy Image

The results of the edge detection algorithms suggest that the content of the image determines the accuracy of the edge detection. Each edge detection technique, in different ways, searches for high frequency components in an image. In the noise-free case, these high frequency components are mostly edges. Unfortunately, this is not true in the noisy case. Assuming the noise is white, there are equal amounts of noise power at each frequency. However, the image has mostly low frequency components, with edges generally not dominating the image. Therefore, when a highpass filter, or any other form of high-frequency detection, is applied to a noisy image, most of the high-frequency components can be characterized by the noise rather than by edges. Consequently, any successful edge detection algorithm quickly deteriorates in performance once noise is added.

NOTE: in the following images, red lines are the edges in the reference mask and green lines are the edges detected by the edge detection filter, but not included in the reference mask.

\section{High-Pass Smoothing Filter}

As the images below show, the high-pass smoothing filter is very consistent in finding the reference mask in the model data. While this edge detection algorithm finds many stray pixels as "edges" in the noisy images, they are not consistent enough to be kept within the reference mask. 


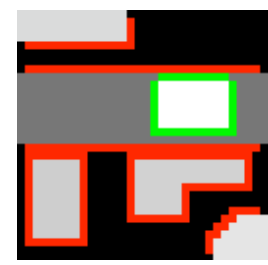

Original Image

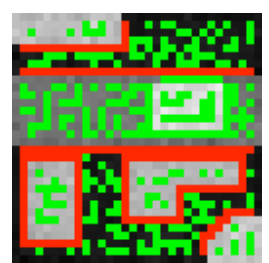

Noisy Image

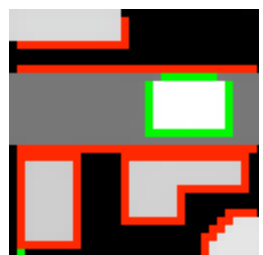

Shifted image

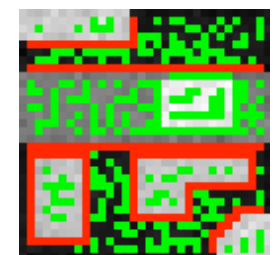

Shifted \& Noisy Image

Unfortunately, this filter is less successful with the real data. Because the edges within the real image are not precisely one pixel wide, the high-pass smoothing filter fails to correctly identify all the edges within the image. When noise is added onto the image, edge detection deteriorates so rapidly that no discernible information can be retrieved.

The ratio of the number of pixels to the number of distinct objects within the image affects the outcome of the edge detection. This is why the edge detection is much more robust on the model image rather than the real data that has many details and few pixels to represent them. The edges on the $32 \times 32$ original image of the street intersection are not successfully detected; whereas, the edges in the $256 \times 256$ resolution image that the $32 \times 32$ image was binned from are accurately detected. This is a result of losing some frequency information when pixel values of the $256 \times 256$ image are averaged to find the new pixel value. The edges detected in each image are shown below.

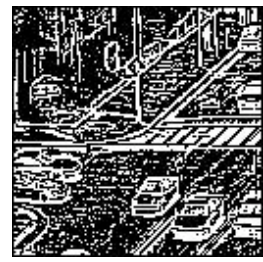

$256 \times 256$ edges

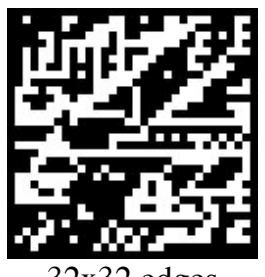

$32 \times 32$ edges

On might question whether it is simply the number of pixels in the image which determine the accuracy of edge detection. This proves to be incorrect when performing the same edge detection algorithm on an up-sampled image of the $32 \times 32$ image. This up-sampled image maintains the same frequency content as the $32 \times 32$ image, but it has $256 \times 256$ pixels. The performance of this same edge detection algorithm did not improve on, simply by increasing the number of pixels.

\section{Ideal High-Pass/Butterworth Filters}

The ideal high-pass filter was not nearly as reliable as the high-pass smoothing filter. While it fairly successfully detected the edges on the original image, the moment noise was added, its performance dropped as can be seen in the images below.

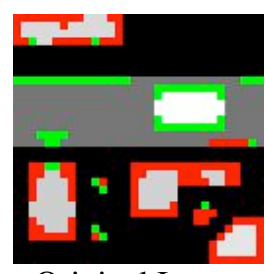

Original Image

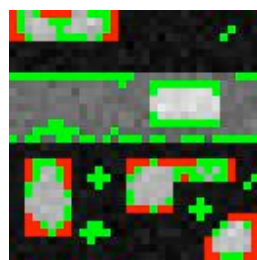

Noisy Image

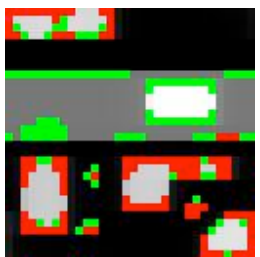

Shifted image

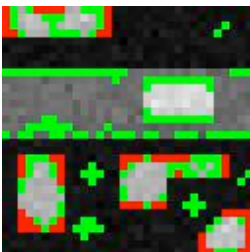

Shifted \& Noisy Image

Again, with the real data, the $32 \times 32$ edge detection results do not reveal any information. In fact, most of the $32 \times 32$ frames of the real data were unable to grab a reference mask because the edges were so few and inconsistent. However, the edge detection from the 256x256, while not as good as the smoothing filter, still accurately exposes the outline of various objects. In the following figure of the edges detected using the ideal high-pass filter on the 256x256 image, at least 3 cars and the crosswalk are apparent. However, in the figure of the edges detected on the 32x32 image, no useful information can be extracted. 


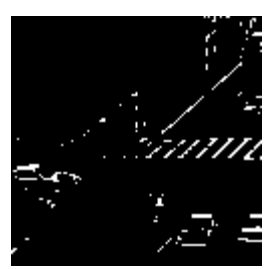

$256 \times 256$ edges

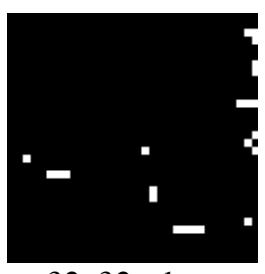

$32 \times 32$ edges

Depending on the image content, edges were detected more or less successfully. Because the cutoff frequency of these filters was hard-coded, some images (e.g. high contrast images, high resolution images), which have distinct high frequency components, will be susceptible to better edge detection than those with lower frequency content. This is one of the reasons edge detection was more successful on the model data with rigid edges than on the real data with blurred edges. Edge detection could be improved by altering the cut-off frequency. However, because the frequency content differs from image to image, there is no "right" cut-off frequency that will work consistently for all, or even most, images. With insufficient knowledge of the frequency content of each image frame, there does not seem to be an efficient and reliable methodology to address the issue the hard-coded cut-off frequency rises.

While the Butterworth filter was slightly more accurate than the ideal high-pass filter at edge detection, it still faced the exact same problems. On real data, these hard-coded high-pass filters fall apart.

\section{Prewitt/Sobel Masks}

Despite the fact that edge masks are implemented in the spatial domain, rather than the frequency domain, edge detection algorithms which use edge masks still face many of the same problems that filters in the frequency domain encountered. Again, while these masks worked quite well for the model data, if there was deviation from single pixel width edges and high contrast images, edge detection would quickly deteriorate. With the presence of noise or with real data that has blurred edges and similar contrast levels between objects, edge masks were no longer able to do edge detection. Edge masks estimate the rate of change between pixel intensity levels. When noise is introduced to these pixel values, the rate of change may not be as large as it was without noise, making the edge more difficult to detect. This is also the case when edges are more than a pixel wide. Without abrupt changes in intensity values, which are generally only achievable in modeled images, edge masks do a poor job of detecting edges.

\section{$2^{\text {nd }}$ Derivative Zero-Crossing}

This method proved to be extremely unsuccessful. Because these are discrete images, the zerocrossings may occur between pixels. The $2^{\text {nd }}$ derivative, zero-crossing edge detection algorithm was altered to take this into account. Edges were defined as pixels whose $2^{\text {nd }}$ derivative was "close to zero" by having the absolute value of the $2^{\text {nd }}$ derivative under an arbitrarily chosen threshold. This resulted in random pixels being deemed edges. This method was by far the worse method of edge detection.

\section{Conclusion}

No edge detection algorithm ever worked consistently. While most edge detection algorithms can be tweaked to work for a single image, none will work for all. With limited prior knowledge of the image content of any subaperature, it is inefficient to adapt each edge detection algorithm to each subaperature.

Image content was a large factor in the success of an edge detection algorithm. While most of the algorithms are likely to be consistent and successful with more pixels, for the application of 
Shack-Hartmann WFS, it is unrealistic to have images with more than $32 \times 32$ pixels. In fact, $16 \times 16$ is more likely. Without a dependable method of detecting edges within each subaperature, it is impractical to use edge detection as a way to isolate moving objects from a static background.

Another issue with using edge detection to detect moving objects that was revealed is that as a moving object goes over part of the reference mask, that part is no longer tracked, even if it returns to the image. This can be addressed by the variance of edge pixels. Each pixel is assigned an indicator random variable defined as 1 if it is an edge, 0 if not. Unfortunately, the variance of these random variables was only helpful in maintaining a consistent reference mask if the edge detection algorithm was already quite accurate.

What did prove to be quite helpful is the variance of each individual pixel value. In time, streets where cars traversed were highlighted by large variances. Even on extremely noisy images where my own eyes could not differentiate moving objects, the variances pinpointed them accurately. Further inquiry into the variance of pixel values will be done to see if this may be a way to isolate moving objects.

\section{References}

[1] Poyneer, L., "Scene-based Shack-Hartmann Wave-Front Sensing: Simulation and Analysis," submitted to Applied Optics

[2] E. Mortensen, "Special Topics: Image Processing and Computer Vision: Lecture 22, Edge Detection”, http://cs.oregonstate.edu/ enm/cs419_519/lectures/lecture22/lecture22.ppt 